\title{
La repercusión de la nueva regulación del horario laboral del Residente sobre la calidad de la enseñanza en el Pregrado y el Postgrado
}

\author{
R.D. Lobato; J. Fernández-Alén y R. Alday
}

Servicio de Neurocirugía. Hospital "12 de Octubre". Madrid.

\section{Resumen}

Se considera la posible influencia negativa de la restricción del horario laboral del residente sobre la enseñanza del pregraduado en los hospitales universitarios. Aunque las opiniones de expertos y los resultados de diferentes estudios sobre la repercusión real de la nueva jornada laboral en la calidad del cuidado clínico y la formación del propio residente son contradictorios, parecen ser más los que indican que su efecto puede ser negativo, particularmente en Europa y Japón, donde el horario semanal quedaría reducido a 48 y 40 horas respectivamente, en clara desventaja con el aplicado en USA, que alcanza 80 horas. EI problema podría agudizarse más aún en España donde la duración de la residencia es más corta que en otros países europeos. Si la formación del residente empeorara sería también de esperar un deterioro añadido en la enseñanza del pregraduado.

PALABRAS CLAVE: Docencia. Jornada laboral. Residente. Estudiante. Pregrado. Postgrado.

The impact of resident work hour limitations on medical student clerkships in Spain

\section{Summary}

The influence of new regulations limiting residents work hours on the total time dedicated and the quality of teaching of medical students in university hospitals is analyzed. Though different studies have shown contradictory results on the possible effects of reduced-hour work week on both patients, safety and resident learning, a great concern is arising in Europe and Japan where duty-hour restriction is much more drastic than in USA (48 and 40 hours vs 80 hours, respectively). Deterioration of residents, training could also diminish the total time dedicated to and quality of medical student education.

Recibido: 15-05-07. Aceptado: 7-06-07
KEY WORDS: Teaching. Duty-hours. Residency. Medical student. Graduate. Postgraduate.

La necesaria complementariedad de las enseñanzas del pregrado y el postgrado en los hospitales adscritos a las Facultades de Medicina podría verse alterada por la próxima reducción del horario laboral del residente, cuya implantación ha de completarse durante el 2008 en todos los países de la $\mathrm{UE}^{23}$. La disminución de la jornada laboral podría afectar no sólo a la formación del residente, sino repercutir también negativamente sobre la calidad de la actividad docente en las rotaciones o estancias clínicas de los alumnos ${ }^{3,5,6,11-18,27}$. En este escrito se considera brevemente la viva polémica existente sobre la pertinencia de la nueva regulación horaria y se comenta la dudosa aplicabilidad de las medidas propuestas para contrarrestar sus posibles efectos negativos sobre la calidad de la docencia médica en su conjunto ${ }^{3,5,6,11,15,18-22}$.

Las principales Instituciones y Agencias que se ocupan de la docencia en Medicina, preconizan que todos los médicos deben ser a la vez aprendices y docentes, y actuar al tiempo como consumidores y proveedores de información a lo largo de su vida profesional. Por ello, desde el inicio de su carrera los estudiantes del pregrado y los jóvenes graduados deben familiarizarse con un amplio rango de técnicas docentes y de aprendizaje, y aceptar el compromiso de enseñar a sus colegas ${ }^{11}$. La enseñanza del pregrado en el siglo XXI se basa en la integración del alumno en la práctica clínica realizada dentro y fuera del ámbito hospitalario, la cual se apoya a su vez de manera sustancial en la actividad asistencial del residente. Diferentes estudios han evidenciado el efecto de fecundación cruzada entre las docencias de pre y postgrado en el continuum ideal que debe vertebrar la enseñanza de la medicina, demostrando, por un lado, que el residente considera un privilegio enseñar al estudiante, porque ello mejora su capacidad de expresarse en público y aumenta su autoestima y satisfacción al sentirse capaz de ayudar a otros; por otro lado, es sabido que los alumnos valoran comparativamente más la enseñanza de los docentes próximos a ellos en edad, que utilizan con mayor soltura las nuevas metodologías de 
acceso y manejo de la información, imprescindibles para el autoaprendizaje y la práctica de la medicina basada en la evidencia. Está claro que adecuadamente programada, la actividad docente del residente proporciona claros beneficios, no sólo al colega junior y al estudiante, sino también a los restantes componentes de la cadena docente, así como al propio paciente y al hospital universitario en el que trabaja $a^{2-5-10,11,18,25}$.

La actividad docente del residente no está normalizada, ni se considera un producto exigible en los hospitales españoles donde se practica de manera incontrolada y heterogénea ${ }^{17}$, no habiéndose determinado ni el grado de aceptación o satisfacción del propio residente con el ejercicio de esta tarea, ni los efectos reales de la misma sobre la formación del residente junior, o del alumno del pregrado. Aunque en los hospitales universitarios los residentes gozan del privilegio teórico de enseñar al colega junior y el estudiante, en ningún sitio figura que tengan la obligación de hacerlo, ni existen mecanismos que controlen o evaluen su grado de dedicación, o incentivos que la motiven. Por ello, sería deseable introducir medidas que faciliten la conjunción y potenciación cruzada de las enseñanzas del pregrado y el postgrado.

El reconocimiento formal del "plus" que conlleva ser residente en un hospital universitario mediante la inclusión de un Adendum en el Programa de Residencia que regulara su dedicación y objetivos docentes en relación con la enseñanza del alumno del Pregrado, junto con la expedición de certificados docentes, o mejor aún, de un nombramiento como profesor colaborador de clases prácticas de los residentes por encima del segundo año, que completaría su currículum y podría acarrear una retribución posterior ligada a la Carrera Profesional, contribuirían sin duda a mejorar la formación del estudiante, y por retroalimentación la del propio residente. Este tipo de nombramiento, que no conlleva retribución económica, pero sí reconocimiento curricular, se ha empezado a otorgar en la Facultad de Medicina de la Universidad Complutense, y podría ser aceptado sin dificultad por la mayoría de las facultades en nuestro país. En cualquier caso, resulta inaceptable que la presencia del alumno no conlleve ningún tipo de compromiso docente explícito, ni ventaja alguna reconocible para el residente que realiza su formación en un hospital universitario. La falta de una definición clara de cual debe ser el perfil y los cometidos docentes de un hospital universitario en nuestro país, y la crónica incongruencia entre las iniciativas docentes de las facultades y los hospitales adscritos a las mismas, explican la escasa o nula atención y dedicación del residente al estudiante de pregrado.

Si la situación de partida en nuestro país parece poco propicia para mejorar la interacción de las docencias del pregrado y el postgrado, el panorama podría empeorar aún más a corto plazo con las nuevas regulaciones del horario laboral del residente implantadas a este y al otro lado del Atlántico, cuyo perfil y posibles implicaciones negativas se comentan a continuación ${ }^{1,4,6,9,13,16,22,23}$.

La reducción del horario laboral de los residentes impuesta por la nueva directiva europea (European Working Time Directive, EWTD ${ }^{23}$ surge pocos años después de un planteamiento similar ocurrido en USA, cuyo objetivo primero fue "proteger al personal médico en formación de la sobrecarga laboral que pueda comprometer el cuidado adecuado de los pacientes". El pistoletazo de salida fue el desenlace del proceso relacionado con la paciente Libby Zion que falleció en 1984 en New York supuestamente como consecuencia de un error de juicio derivado de la fatiga y la deprivación de sueño de los residentes que la atendieron. Tras un prolongado debate, el Accreditation Council for Graduate Medical Education (ACGME) implementó en Julio del 2003 una normativa que limita el horario laboral del residente a 80 horas semanales, estableciendo además los intervalos de distribución de estas horas con el propósito esencial de garantizar una atención más segura y eficiente del paciente, y mejorar la disponibilidad de tiempo para el estudio y el aprendizaje del residente, libre ya de la fatiga que experimentaba con el anterior sistema de formación iniciado a principios del siglo pasado en el Johns Hopkins, y que exigía al residente una vida "sacerdotal" (house officer) sin limitación horaria alguna. Esta misma idea ha llevado también a las autoridades de la Unión Europea y el Japón a reducir drásticamente el horario laboral del residente.

La nueva directiva europea, que impone alcanzar el límite de 48 horas semanales el próximo año 2008, regula que el residente trabajará 8 horas al día, 6 días por semana, incluyendo el tiempo dedicado a guardias nocturnas ${ }^{23}$. La violación de esta normativa se penaliza con 15.000 euros, $\mathrm{y}$ en USA, a la sanción económica, puede añadirse la retirada de la venia docente. Sin embargo, se ha comprobado que en Europa sólo el 50\% de los centros la cumplen, y que en realidad se realiza una notable cantidad de trabajo extra para continuar asegurando una buena atención al paciente; así, esta "aportación laboral voluntaria" es tolerada tácitamente, pero no se comunica para evitar sanciones, habiéndose generado ya un "área gris de ilegalidad" en muchos países de nuestro entorno.

El aprendizaje del residente se basa en el equilibrio entre los requerimientos de su formación teórico-practica y la realización del trabajo asistencial, por lo que en caso de conflicto de disponibilidad horaria entre estos dos ingredientes, el residente se verá forzado a dedicar proporcionalmente más tiempo a la asistencia en su vertiente precisamente más rutinaria, o menos formativa. Así, la obligación de realizar la misma labor asistencial en un horario mas reducido, recortará tanto sus actividades clínicas, como el tiempo total dedicado a la docencia del resi- 
dente junior y del estudiante del pregrado. Por otra parte, la asunción de parte del trabajo rutinario del residente por parte del staff con compromiso docente, menoscabará la disponibilidad y dedicación de este profesorado para la enseñanza del estudiante y del propio residente. Además, la calidad del cuidado del paciente, que determina a su vez la calidad de la actividad docente, se resentirá debido a la ruptura de continuidad en la atención clínica y la disminución de la experiencia clínica total del residente.

Los profesionales en Europa reconocen que el antiguo horario laboral de 80-100 horas semanales era excesivo (recordemos que en USA el de 80 horas no se considera tal), pero la mayoría de los médicos y cirujanos experimentados, así como los residentes senior, consideran el limite de 48 horas excesivamente precario para alcanzar un nivel de competencia adecuado al final de la residencia. La polémica sobre los efectos de la restricción horaria en la formación del residentes y alumnos de pregrado está pues servida, pero no resuelta.

La cuestión, tanto en USA como en Europa y Japón (país este último donde la restricción horaria se desploma hasta 40 horas semanales), es si la reducción del horario laboral mejora realmente la atención del paciente y las oportunidades de formación del residente. En USA ya se ha comprobado que, aparte del consecuente incremento de los costes asistenciales ligados a la contratación de médicos del staff para asumir la disminución de la fuerza laboral proporcionada antes por el residente, el recorte horario no garantiza necesariamente la consecución de dichos objetivos, por lo que se reclama insistentemente por docentes y residentes una grado mayor de flexibilidad en la aplicación da la restricción horaria, particularmente en el caso del residente senior, que debe apurar al máximo el cumplimiento de su formación en el momento en que su aprendizaje es más cualificado ${ }^{5,9,17,20,23}$. Así, y ante las presiones ejercidas desde el mundo de la medicina académica, la ACGME ha aceptado ampliar el horario semanal de los residentes senior de especialidades quirúrgicas de 80 a 88 horas.

Aparte de las opiniones contrapuestas sobre las ventajas y desventajas de la nueva regulación horaria entre directores de programas de formación, expertos en docencia médica, sociedades médicas, y los propios residentes, los resultados de diferentes estudios aportan conclusiones también contradictorias, y así mientras algunos indican que la atención al enfermo no se ha deteriorado, al tiempo que el grado de satisfacción del residente con su nueva vida laboral se ha incrementado, son más los que han detectado que el trabajo clínico por turnos, que conlleva una pérdida de continuidad en la atención y seguimiento del enfermo, menoscaba tanto el cuidado del paciente, como el aprendizaje del residente $4,6,9,13,15,16,21,22,24$. En la medida en que esto sea cierto, sus efectos negativos resultaran más dramáticos en Europa y Japón, donde muchos expertos afirman que resultará imposible formar residentes con el nivel alcanzado hasta ahora; esta imposibilidad podría ser absoluta en el caso de las especialidades quirúrgicas, en las que la experiencia clínica y operatoria del residente se reducirá en un $30-35 \%$ durante los 6 años preceptivos de formación de los actuales programas, situación que se agudizará más aún en España donde la duración de las residencias está limitada a 5 años $^{17}$.

Como se especifica en el Position Paper del EWTD ${ }^{23}$, tras aplicar el régimen laboral de 48 horas semanales el número de horas anuales trabajadas por los residentes europeos descenderá de 3760 a 2256 (reducción del 40\% del tiempo total de trabajo), y su presencia efectiva se reducirá de 225 días por año a tan solo 150 días. De acuerdo con dicho documento esta limitación del horario laboral puede conllevar las siguientes consecuencias negativas: 1. Reducción considerable del número de pacientes vistos por el residente durante su formación (disminución del $30-40 \%$ de participación en operaciones quirúrgicas); 2. La adquisición de la información sobre los pacientes y el seguimiento completo de su historia desde la admisión al alta, se verá limitada; así, un residente quirúrgico que lleva a un paciente puede no participar en la operación del mismo, o en el seguimiento inmediato postoperatorio; 3. Disrupción repetitiva de la relación formativa del residente con sus tutores, que será más breve y errática, o menos estructurada; 4. Los residentes tendrán una sobrecarga proporcionalmente mayor de trabajo "rutinario", y el tiempo para enseñanza programada y reglada, y la asistencia a sesiones, seminarios y otros encuentros docentes disminuirá notablemente; 5 . La disponibilidad del residente para le enseñanza del pregraduado se verá mermada, y 6 . Finalmente, la formación en investigación pasara a ser un "hobby" y desaparecerá de hecho de los programas de residencia en los que actualmente figura, con la excepción otra vez de España, donde los programas nunca han incluido rotación por laboratorio o área de investigación ${ }^{17}$.

Las consecuencias para la atención de los pacientes podrían resultar no menos negativas. En la mayoría de los servicios será inevitable adoptar, o bien un sistema de turnos en los que los periodos fuera de hora normal se cubrirán por turnos de 16 a 24 horas ("partial shift system"), o bien a un sistema de turno rígido ("full shift"), en el que el horario se divide en bloques rígidos con médicos adscritos a cada turno. Este cambio requiere del traspaso de información acerca de la situación del paciente entre turnos, ejercicio que conlleva riesgos demostrados para su seguridad. Para solucionar este problema se han propuesto medidas como el "night-float" en USA, o la del médico de guardia no residente ("non-resident on call") con la que residentes jóvenes, o de primeros años, cubren las noches; sin embargo, esto resulta peligroso para el paciente complejo, y por tanto es inaceptable en las especialidades quirúrgicas. Por otra 
parte, estos sistemas conducen a la despersonalización de la atención, y crean una mentalidad de turno en el residente, que desde un punto de vista clínico general no es mejor que la antigua ${ }^{1,23}$ y que posiblemente altere también la continuidad en la atención al estudiante.

Para contrarrestar los problemas mencionados se han propuesto diferentes alternativas, la mayoría de las cuales resultan, sin embargo, difíciles, o incluso imposibles de aplicar en la vida real ${ }^{23}$. Entre ellas estarían: a) La reducción del trabajo burocrático de los residentes; si bien se ha demostrado en algunos hospitales que un staff no médico puede asumir con éxito muchas de estas labores, (informes diversos, consentimientos, etc), los administradores son contrarios a crear un nuevo cuerpo laboral por el consecuente aumento de los costes. b) Incrementar en un 10-15\% el número de residentes o médicos de staff; sin embargo, esta medida, aparte de conllevar un coste adicional inaceptable, sobrecargaría a los profesores disponibles y generaría una competitividad perversa por el número de pacientes y operaciones disponibles por residente; por otra parte, el número de especialistas se incrementaría por encima de las necesidades reales, colmadas ya en la mayoría de los países (aparecería la inflación de especialistas). c) Contrarrestar los defectos en la formación aumentado el numero de sesiones, seminarios, cursos, lecciones, etc; esta medida, útil en especialidades médicas, no lo sería del todo en las quirúrgicas, en las que el número de operaciones es crítico para lograr una buena formación, y d) Prolongar la duración de la residencia en 1 ó 2 años, lo que requeriría aumentar el tiempo total de la formación médica de 16 a 18 años con la consiguiente desmotivación para elegir esta carrera.

Al considerar totalmente insuficiente el límite de 48 horas semanales para completar una formación adecuada del residente, los responsables de las especialidades quirúrgicas de la Unión Europea, que integran a mas de 80.000 especialistas, (Secciones de Cirugía de la UEMS) han propuesto la modificación de la normativa de la EWTD mediante la opción llamada " $48+12$ horas", que añade al horario oficial 12 horas reservadas y protegidas para actividades formativas y docentes, estructuradas y supervisadas, pero nunca dedicadas a guardias nocturnas o trabajo rutinario ${ }^{23}$. La cuestión es qué porcentaje de este tiempo ya escaso, estaría dispuesto a dedicar el residente a la enseñanza y monitorización del alumno de pregrado, y parece fácil predecir que en el mejor de los casos la atención empeoraría.

Unos pocos estudios recientes sobre la percepción de los propios estudiantes acerca de la disponibilidad horaria del residente, y el grado de dedicación e interés de éste por la enseñanza del pregrado medidos antes y después de la aplicación de la restricción horaria, indica que no se produjeron efectos o cambios negativos ${ }^{19,27}$, o que éstos sólo ocurren durante las rotaciones por unidades quirúrgicas ${ }^{19}$. Esta aparente paradoja se atribuyó al beneficio derivado de la disminución de la fatiga del residente y la reducción compensada del trabajo "rutinario" que realizaba, el cual pasó a cargo de otro personal (enfermeras y otros). Sin embargo, hay que enfatizar que estos análisis se realizaron en hospitales que implantaron medidas y realizaron inversiones en nuevos recursos docentes específicamente destinadas a contrarrestar la disminución del horario laboral del residente y mantener el nivel de la calidad educativa. Por ello, estas impresiones no pueden extrapolarse a la gran mayoría de los hospitales, los nuestros incluidos, en los que tales medidas no van a ser arbitradas por su alto coste, a menos que la propia sociedad y las autoridades político-sanitarias decidieran lo contrario.

Por ello, la cuestión inicial recurre y uno vuelve a preguntarse si el nuevo régimen laboral, tan restrictivo en Europa, permitirá al residente, obsesionado ya con su propia formación, participar eficazmente en la enseñanza del pregrado. Aunque medidas tales como la aplicación de nuevas tecnologías de la enseñanza, el uso sistemático de los simuladores, la mejora de la formación especifica de los profesores ("train the trainers"), etc, pueden contrarrestar parte de los efectos negativos de la restricción horaria, nadie sabe en qué medida va a resultar finalmente afectada la enseñanza del pregraduado en los hospitales universitarios, amenazada ya como está por la complejidad creciente de las patologías de los pacientes ingresados y las presiones continuas para acortar sus estancias, que impiden al alumno contemplar el curso global de los procesos patológicos más comúnmente tratados por el médico general.

El problema se podría paliar desplazando al estudiante al contexto de la medicina ambulatoria durante un periodo importante de su formación. Esta medida, que se extiende cada vez más en los países desarrollados, no es un mal menor, porque el contacto con el enfermo ambulatorio permite al alumno realizar un seguimiento más completo y próximo de las enfermedades que debe conocer y manejar un generalista, que es para lo que se le debe formar. Son más cada vez los estudios que demuestran la satisfacción del alumno y la calidad de su formación global cuando recibe entrenamiento en la medicina extrahospitalaria ${ }^{7,8}$.

\section{Bibliografía}

1. Barone, J.E., Ivy, M.E.: Resident work hours: The five stages of grief. Acad Med 2004; 79: 379-380

2. Barzansky, B., Etzel, S.I.: Educational Programs in US Medical Schools, 2003-2004. JAMA 2004; 292: 1025-1031

3. Calman, K.G., Temple, J.G., Naysmith, R., et al.: Reforming higher specialist training in United Kingdom. A step along the continuum of medical education. Medical Education 1999; 33: 28-33.

4. Cohen-Gadol, A.A., Piepgras, D.G., Krishnamurthy, 
S., Fessler, R.D.: Resident duty hours reform. Results of a national survey of the Program Directors and Residents in Neurosurgey Training Programs. Neurosurgery 2005; 56: 398-403.

5. Coverdill, J.E., Finlay, W., Adrales, G.L., et al.: Dutyhours restrictions and the work of surgical faculty: results of a Multi-Institutional study. Acad Med 2006; 81: 50-56.

6. Charap, M.: Reducing resident work hours: unproven assumptions and unforeseen outcomes. Ann Intern Med 2004; 140: 814-815.

7. Dent, J.A., Angell-Preece, H.M., Ball, H.M.L., Ker, J.S.: Using the ambulatory care teaching centre to develop opportunities for integrated learning. Medical Teacher 2001; 23: 171-175.

8. Ewart, B., Sandars, J.: Community involvement in undergraduate medical education. Clinical Teacher 2006; 3 : 148-153.

9. Friedman, W.A.: Resident duty hours in American Neurosurgery. Neurosurgery 2004; 54: 925-933.

10. Gaba, D.M., Howard, S.K.: Fatigue among clinicians and the safety of patients. N Eng J Med 2002: 347: 12491255 .

11. GMC. Tomorrow,s Doctors, 2003. http://www.gmcuk.org.

12. Gough, J., Beckett, D.: Young doctors as a teachers: understanding their motivations and needs. Clinical Teacher 2006; 3: 49-52.

13. Jagsi, R., Shapiro, J., Weinstein, D.F.: Perceived impact of resident work hour limitations on medical student clerkships: A survey study. Acad Med 2005: 80: 752-757.

14. Kogan, J.R., Bellini, L.M., Shea, J.A.: Forces impacting graduate medical education. Acad Med 2004; (Suppl) 79: S58-S61.

15. Lin, G.A., Beck, D.C., Garbutt, J.M.: Residents, perception of the effect of work hours limitations at large teaching hospital. Acad Med 2006; 81: 63-67.

16. Lindsay, K.W.: Neurosurgical training in the United Kingdom and Ireland: Assesssing progress and attainment. Neurosurgery 2002; 50: 1103-1113.

17. Lobato, R.D., Fernández Alén, J., Alday, R., Gómez,
P.A., Lagares, A.: El nuevo Programa de Residencia en Neurocirugía en España. Neurocirugía 2003; 14: 281-294.

18. London Deanery. Teaching and learning in clinical contexts: A resource for health professionals http://www.clin icalteaching.nhs.uk .

19. Papp, K.K., Stoller, E.P., Sage, P., et al.: The effects of sleep loss and fatigue on resident-physicians: A multi-institutional, mixed-method study. Acad Med 2004; 79: 394-406.

20. Pellegrini, C.A., Warshaw, A.L., Debas, H.T.: Residency training in surgery in the $21^{\text {th }}$ century. A new paradigm. Surgery 2004; 136: 953-965.

21. Romanchuk, K.: The effect of limiting residents, work hours on their surgical training: A Canadian perspective. Acad Med 2004; 79: 384-385.

22. Steinbrook, R.: The debate over residents, work hours. N Engl J Med 2002; 347: 1296-1302.

23. The European Working Time Directive and the Effects on Training of Surgical Specialists (Doctors in Training). Acta Neurochir (Wien) 2006; 148: 1130.1136.

24. Veasey, S., Rosen, R., Barzansky, B., et al.: Sleep loss and fatigue in residency training: a reappraisal. JAMA 2002; 288: 1116-1124.

25. Weinstein, D.F.: Duty hours for resident physicianstough choices for teaching hospitals. N Eng J Med 2002; 347: 1275-1278.

26. Whitcomb, M.E.: More on resident duty-hours limit. Acad Med 2004; 79: 377-378.

27. White, C.B., Haftel, H.M., Purkiss, J.A., Schigelone, A.S., Hammoud, M.M.: Multidimensional effects of the 80hour work week at the University of Michigan medical school. Acad Med 2006; 81: 57-62.

Lobato, R.D.; Fernández-Alén, J.; Alday, R.: La repercusión de la nueva regulación del horario laboral del Residente sobre la calidad de la enseñanza en el Pregrado y el Postgrado. Neurocirugía 2008; 19: 213-217.

Correspondencia postal: Ramiro D. Lobato, Servicio de Neurocirugía. Hospital "12 de Octubre". Avda Córdoba, s/n, 28041, Madrid. 